\title{
Erratum
}

\section{Erratum: Steinkellner et al., Amphetamine Action at the Cocaine- and Antidepressant-Sensitive Serotonin Transporter Is Modulated by $\alpha$ CaMKII}

In the article "Amphetamine Action at the Cocaine- and Antidepressant-Sensitive Serotonin Transporter Is Modulated by $\alpha$ CaMKII" by Thomas Steinkellner, Therese R. Montgomery, Tina Hofmaier, Oliver Kudlacek, Jae-Won Yang, Mattias Rickhag, Gangsoo Jung, Gert Lubec, Ulrik Gether, Michael Freissmuth, and Harald H. Sitte, which appeared on pages 8258-8271 of the May 27, 2015 issue, there was a mix-up in author affiliations. The corrected author list and affiliations are as follows: Thomas Steinkellner, ${ }^{1}$ Therese R. Montgomery, ${ }^{1,2}$ Tina Hofmaier, ${ }^{1}$ Oliver Kudlacek, ${ }^{1}$ Jae-Won Yang, ${ }^{1}$ Mattias Rickhag, ${ }^{3}$ Gangsoo Jung, ${ }^{4}$ Gert Lubec, ${ }^{4}$ Ulrik Gether ${ }^{3}$ Michael Freissmuth, ${ }^{1}$ and Harald H. Sitte. ${ }^{1,5}$ Their affiliations are as follows: ${ }^{1}$ Institute of Pharmacology, Center for Physiology and Pharmacology, Medical University of Vienna, 1090 Vienna, Austria, ${ }^{2}$ UCD Conway Institute of Biomolecular and Biomedical Research, University College Dublin, Belfield, Dublin 4, Ireland, ${ }^{3}$ Molecular Neuropharmacology and Genetics Laboratory, Department of Neuroscience and Pharmacology, Faculty of Health and Medical Sciences, The Panum Institute, University of Copenhagen, Denmark, ${ }^{4}$ Department of Pediatrics, Medical University of Vienna, 1090 Vienna, Austria, and ${ }^{5}$ Center for Addiction Research and Science, Medical University of Vienna, 1090 Vienna, Austria. The author line and affiliations have been corrected on the online PDF version.

DOI: $10.1523 / J N E U R O S C I .2730-15.2015$ 